Rev. Inst. Flor. v. 26 n. 1 p. 101-109 2014

http://dx.doi.org/10.4322/rif.2014.007

ISSN impresso 0103-2674/on-line 2178-5031

\title{
FLORA PTERIDOFÍTICA DE FRAGMENTOS FLORESTAIS DO PARQUE ESTADUAL DE VASSUNUNGA - SANTA RITA DO PASSA QUATRO, SP ${ }^{1}$

\author{
PTERIDOPHYTE FLORA OF FOREST FRAGMENTS IN \\ VASSUNUNGA STATE PARK - SANTA RITA DO PASSA QUATRO, SÃO PAULO, BRAZIL
}

\author{
Áurea Maria Therezinha COLLI²; Sonia Aparecida de SOUZA-EVANGELISTA2,3; \\ André Luís Teixeira de LUCCA²
}

\begin{abstract}
RESUMO - Este trabalho apresenta o levantamento da flora pteridofítica de dois fragmentos florestais do Parque Estadual de Vassununga, unidade de conservação localizada no município de Santa Rita do Passa Quatro, SP. Para tanto, foram realizadas onze excursões por caminhos e trilhas de fiscalização e de pesquisa. Na Gleba Maravilha, que apresenta vegetação de Floresta Estacional Semidecidual Montana e Floresta Estacional Semidecidual Aluvial com inundação temporária, foram encontradas 10 famílias, 18 gêneros e 35 espécies, sendo Pteridaceae, Thelypteridaceae, Aspleniaceae, e Polypodiaceae as famílias mais ricas com nove, sete, seis e cinco espécies, respectivamente. Na Gleba Capão da Várzea, onde ocorre a Floresta Paludosa e o Campo Antrópico, ocorreram nove famílias, 14 gêneros e 24 espécies, onde Thelypteridaceae e Polypodiaceae se apresentaram como as mais ricas, com oito e seis espécies, respectivamente. As Glebas do Parque Estadual de Vassununga com as suas diferentes fisionomias vegetais são áreas importantes para a conservação de pteridófitas na região nordeste do Estado de São Paulo, principalmente as típicas de Floresta Estacional Semidecidual Montana e de Floresta Paludosa como Blechnum brasiliense, Campyloneurum angustifolium, Cyclodium meniscioides, Osmunda regalis, Pityrogramma calomelanos e Thelypteris opposita.
\end{abstract}

Palavras-chave: Pteridófitas; Floresta Estacional Semidecidual; Floresta Paludosa.

\begin{abstract}
This work presents a survey on pteridophyte flora of two forest fragments in Vassununga State Park, a conservation unit located in the city of Santa Rita do Passa Quatro, São Paulo, Brazil. In order to carry out the survey, a series of eleven tours on old inspection and research trails were made. In the Maravilha Tract, which consists of Seasonal Semi-Deciduous Montane Forest and Seasonal Semideciduous Alluvial Forest with temporary flooding, 10 families, 18 genera, and 35 species were found, the richest families were Pteridaceae, Thelypteridaceae, Aspleniaceae, and Polypodiaceae, with nine, seven, six and five species, respectively. In the Capão da Várzea Tract, which is dominated by Swamp Forest, a total of nine families, 14 genera, and 25 species were found, the richest families were Thelypteridaceae and Polypodiaceae with eight and six species, respectively. The tracts in Vassununga State Park have a diverse vegetation and are important for the conservation of pteridophytes in northeastern São Paulo, in particular of those typical of Seasonal Semideciduous Forest and Swamp Forest, such as Blechnum brasiliense, Campyloneurum angustifolium, Cyclodium meniscioides, Osmunda regalis, Pityrogramma calomelanos, and Thelypteris opposita.
\end{abstract}

Keywords: Pteridophytes; Seasonal Semideciduous Forest; Swamp Forest.

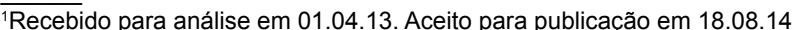

${ }^{2}$ Instituto Florestal, Rua do Horto, 931, 02377-000 São Paulo, SP, Brasil.

${ }^{3}$ Autor para correspondência: Sonia Aparecida de Souza-Evangelista - soniasouza@if.sp.gov.br 


\section{INTRODUÇÃO}

As pteridófitas são componentes abundantes da flora e fundamentais ao estabelecimento de outros grupos vegetais e animais; habitam diversos microambientes, além de se constituirem em testemunhos de características passadas e atuais de uma região (Barros e Costa e Silva, 1996; Prado, 1998).

No Brasil, ocorrem 1.176 espécies de pteridófitas e o Estado de São Paulo é o segundo mais diverso do país, com 45 espécies de licófitas e 522 de samambaias (Prado e Silvestre, 2010; Prado e Hirai, 2012).

A Mata Atlântica sensu lato é a formação vegetal brasileira que abriga o maior número de pteridófitas. No Estado de São Paulo, a maioria das espécies é encontrada ao longo da Serra do Mar, da Mantiqueira e no Vale do Ribeira (Prado, 2007).

Estudos sobre a flora pteridofítica no interior do Estado de São Paulo foram realizados a partir da década de 1990 por Windisch (1992), Salino (1993; 1996), Prado (1998), Siqueira e Windisch (1998), Colli et al. (2003), Colli et al. (2004a, b, c), Colli et al. (2007) e por Nóbrega e Prado (2008) em áreas com vegetação de Floresta Estacional Semidecidual, Mata Ciliar, Cerrado e Floresta Paludosa.

A Floresta Estacional Semidecidual, as matas ciliares e de brejo dos municípios de Analândia, Itirapina, Brotas, Porto Ferreira e Santa Rita do Passa Quatro apresentam maior riqueza de pteridófitas em relação ao Cerrado (Salino, 1993; 1996, Colli et al., 2003, Colli et al., 2004a, b, c) ; enquanto no Jardim Botânico de Bauru, a maior diversidade ocorreu nas matas de brejo e no Cerrado.

Nesse contexto, o presente trabalho teve o objetivo de realizar o levantamento de pteridófitas nos fragmentos florestais do Parque Estadual de Vassununga - glebas Maravilha e Capão da Várzea - e contribuir com o conhecimento da diversidade do grupo em remanescentes de Floresta Estacional Semidecidual, Mata Ciliar e Floresta Paludosa na região nordeste do Estado de São Paulo.

\section{MATERIAL E MÉTODOS}

O Parque Estadual de Vassununga é uma das unidades de conservação do interior do Estado de São Paulo, localiza-se no município de Santa Rita do Passa Quatro - SP, com área total de 2.071,42 hectares, composto por seis fragmentos independentes: Glebas Praxedes, Pé-de-Gigante, Capetinga Oeste, Capetinga Leste, Capão da Várzea, e Maravilha (Figura 1), sob as coordenadas $21^{\circ} 41^{\prime} 00^{\prime}$ - " $21^{\circ} 41$ '21" S e 4734'37" - 47'39'39" W6 (Ribeiro, 2009).

O clima da região, segundo a classificação de Köppen é do tipo Cwa, quente de inverno seco com temperaturas aproximadas de $18^{\circ} \mathrm{C}$ no inverno e superiores a $22{ }^{\circ} \mathrm{C}$ no verão, e média anual de $23,3{ }^{\circ} \mathrm{C}$, em que a precipitação varia de 1.300 a $1.700 \mathrm{~mm}$.

A divisão dos estudos de pteridófitas por glebas, nesse Parque, foi necessária devido à localização, distância entre as mesmas e às diferentes fisionomias vegetais.

O presente estudo foi realizado na gleba Maravilha, com área de 131,52 hectares, localizada à margem direita do rio Mogi Guaçu, onde predomina a vegetação de floresta Estacional Semidecidual Montana e Floresta Estacional Semidecidual Aluvial com inundação temporária, entre as coordenadas $21^{\circ} 44^{\prime} 01^{\prime \prime} \mathrm{S}-4^{\circ} 39^{\prime} 01^{\prime \prime} \mathrm{W}$ e na gleba Capão da Várzea, com 12,18 hectares e vegetação de Floresta Estacional Semidecidual aluvial de inundações permanentes - Floresta Paludosa e Campo Antrópico, localizada em uma planície de inundação da bacia hidrográfica do rio Mogi-Guaçu, entre as coordenadas 21 ${ }^{\circ} 43^{\prime} 51^{\prime \prime} \mathrm{S}-47^{\circ} 36^{\prime} 43^{\prime \prime} \mathrm{W}^{6}$. 


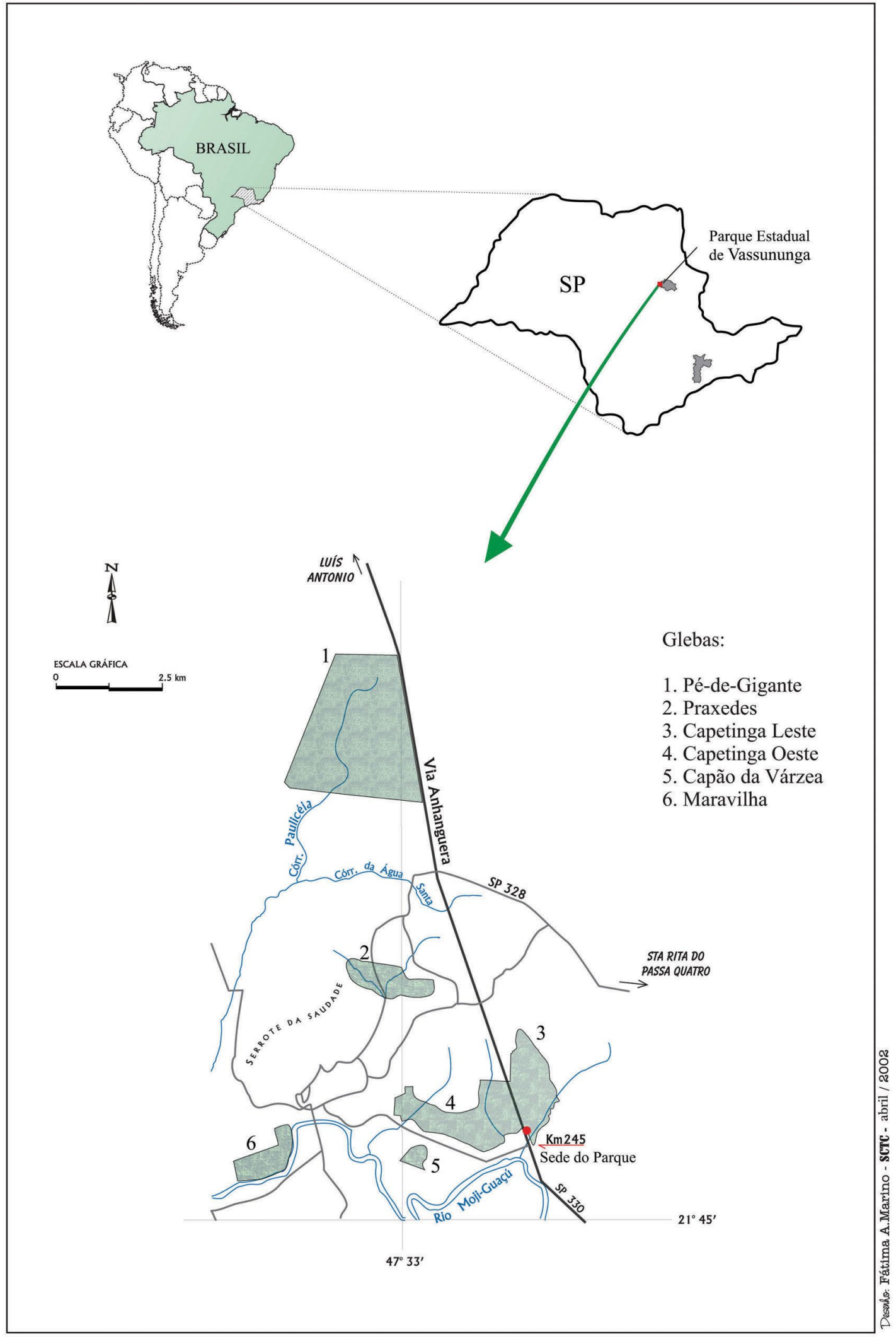

Figura 1. Mapa de localização do Parque Estadual de Vassununga. Glebas Capão da Várzea (5) e Maravilha (6).

Figure 1. Map with the location of Vassununga State Park. Capão da Várzea (5) and Maravilha (6) tracts.

Rev. Inst. Flor. v. 26 n. 1 p. 101-109 jun. 2014 
Para o levantamento de pteridófitas foram realizadas onze excursões, sendo oito entre os meses de fevereiro a dezembro de 2004, na gleba Maravilha, e três entre os meses de janeiro a junho de 2005, na gleba Capão da Várzea. Em ambas as áreas foram utilizados caminhos e trilhas de fiscalização e de pesquisa antigos.

Algumas espécies foram identificadas por comparação com materiais depositados no Herbário do Instituto Florestal - SPSF e outras por especialistas. Para verificar o status de conservação foi consultada a Resolução SMA $\mathrm{n}^{\circ}$ 48, de 21/9/2004 e o Livro Vermelho das Espécies Vegetais Ameaçadas de São Paulo (Prado, 2007).
A classificação das espécies foi revista a partir do Checklist das licófitas e samambaias do Estado de São Paulo (Prado e Hirai, 2012).

\section{RESULTADOS E DISCUSSÃO}

Nesse estudo foram identificados o total de 13 famílias, 23 gêneros e 51 espécies. Na gleba Maravilha ocorreram 10 famílias, 23 gêneros e 35 espécies e na Capão da Várzea nove famílias, 14 gêneros e 25 espécies de pteridófitas, conforme apresenta a Tabela 1.

Tabela 1. Lista das pteridófitas das glebas Maravilha e Capão da Várzea do Parque Estadual de Vassununga. Hábito TE $=$ terrestre, $\mathrm{EP}=$ epifítico. Ambiente $\mathrm{MC}=$ Mata Ciliar, FES $=$ Floresta Estacional Semidecidual, $\mathrm{FP}=\mathrm{Floresta}$ Paludosa, $\mathrm{CA}=$ Campo Antrópico. 0 = ausência e 1 = presença.

Table 1. List of pteridophytes in the Maravilha and Capão da Várzea tracts within Vassununga State Park. $\mathrm{TE}=$ terrestrial; $\mathrm{EP}=$ epiphyte; $\mathrm{MC}=$ Riparian Forest; FES = Seasonal Semideciduous Forest; FP = Swamp Forest; $\mathrm{CA}=$ Man-made Grassland. $0=$ absence; $1=$ presence.

\begin{tabular}{|c|c|c|c|c|c|c|c|c|}
\hline Família/Espécie & $\mathrm{TE}$ & EP & $\mathrm{MC}$ & FES & FP & CA & Maravilha & $\begin{array}{c}\text { Capão } \\
\text { da } \\
\text { Várzea }\end{array}$ \\
\hline ANEMIACEAE & 1 & 0 & 1 & 0 & 0 & 0 & 1 & 0 \\
\hline Anemia phyllitidis (L.) Sw. & 1 & 0 & 1 & 0 & 0 & 0 & 1 & 0 \\
\hline ASPLENIACEAE & 1 & 0 & 1 & 1 & 1 & 0 & 1 & 1 \\
\hline Asplenium auritum $\mathrm{Sw}$. & 1 & 0 & 1 & 1 & 0 & 0 & 1 & 0 \\
\hline Asplenium claussenii Hieron. & 1 & 0 & 1 & 1 & 0 & 0 & 1 & 0 \\
\hline Asplenium cristatum Lam. & 1 & 0 & 0 & 1 & 0 & 0 & 1 & 0 \\
\hline Asplenium inaequilaterale Willd. & 1 & 0 & 1 & 1 & 0 & 0 & 1 & 0 \\
\hline Asplenium otites Link & 1 & 0 & 0 & 1 & 0 & 0 & 1 & 0 \\
\hline Asplenium sp. & 1 & 0 & 0 & 0 & 1 & 0 & 0 & 1 \\
\hline Asplenium squamosum L. & 1 & 0 & 1 & 1 & 0 & 0 & 1 & 0 \\
\hline BLECHNACEAE & 1 & 0 & 1 & 0 & 1 & 0 & 1 & 1 \\
\hline Blechnum brasiliense Desv. & 1 & 0 & 0 & 0 & 1 & 0 & 0 & 1 \\
\hline Blechnum serrulatum Rich. & 1 & 0 & 1 & 0 & 0 & 0 & 1 & 0 \\
\hline CYATHEACEAE & 1 & 0 & 0 & 1 & 1 & 0 & 0 & 1 \\
\hline Cyatehea delgadii Sternb. & 1 & 0 & 0 & 1 & 1 & 0 & 0 & 1 \\
\hline DENNSTAEDTIACEAE & 1 & 0 & 0 & 0 & 0 & 1 & 0 & 1 \\
\hline Pteridium aquilinum (L.) Kuhn & 1 & 0 & 0 & 0 & 0 & 1 & 0 & 1 \\
\hline
\end{tabular}


continuação - Tabela 1

continuation - Table 1

\begin{tabular}{|c|c|c|c|c|c|c|c|c|}
\hline Família/Espécie & $\mathrm{TE}$ & $\mathrm{EP}$ & MC & FES & FP & $\mathrm{CA}$ & Maravilha & $\begin{array}{c}\text { Capão } \\
\text { da } \\
\text { Várzea }\end{array}$ \\
\hline DRYOPTERIDACEAE & 1 & 0 & 1 & 1 & 1 & 0 & 1 & 1 \\
\hline Bolbitis serratifolia (Mert. ex Kaulf.) Schott & 1 & 0 & 1 & 1 & 0 & 0 & 1 & 0 \\
\hline Cyclodium meniscioides (Willd) C. Presl. & 1 & 0 & 0 & 1 & 1 & 0 & 0 & 1 \\
\hline Ctenitis distans (Brack.) Ching & 1 & 0 & 0 & 0 & 1 & 0 & 0 & 1 \\
\hline Ctenitis submarginalis (Langsd. \& Fisch.) Ching & 1 & 0 & 1 & 1 & 0 & 0 & 1 & 1 \\
\hline Lastreopsis effusa (Sw.) Tindale & 1 & 0 & 1 & 1 & 0 & 0 & 1 & 0 \\
\hline LOMARIOPSIDACEAE & 1 & 0 & 1 & 0 & 0 & 0 & 1 & 0 \\
\hline Nephrolepis cordifolia (L.) C.Presl & 1 & 0 & 1 & 0 & 0 & 0 & 1 & 0 \\
\hline OSMUNDACEAE & 1 & 0 & 0 & 1 & 0 & 0 & 0 & 1 \\
\hline Osmunda regalis L. var. spectabilis (Willd.)A.Gray & 1 & 0 & 0 & 1 & 0 & 0 & 0 & 1 \\
\hline POLYPODIACEAE & 1 & 1 & 1 & 1 & 1 & 0 & 1 & 1 \\
\hline Campyloneurum angustifolium (Sw.) Fée & 1 & 0 & 0 & 1 & 0 & 0 & 0 & 1 \\
\hline Campyloneurum major (Hieron ex. Hicken) Lellinger & 1 & 0 & 1 & 1 & 0 & 0 & 1 & 0 \\
\hline Campyloneurum minus Fée & 1 & 0 & 0 & 1 & 0 & 0 & 1 & 0 \\
\hline Microgramma lindbergii (Mett. ex Kuhn) de la Sota & 0 & 1 & 1 & 1 & 0 & 0 & 1 & 0 \\
\hline Microgramma sp. & 0 & 1 & 0 & 1 & 0 & 0 & 0 & 1 \\
\hline Pleopeltis pleopeltifolia (Raddi) Alston & 0 & 1 & 1 & 1 & 0 & 0 & 1 & 1 \\
\hline Pleopeltis astrolepis (Liebm.) E.Fourn. & 0 & 1 & 0 & 1 & 1 & 0 & 0 & 1 \\
\hline Polypodium fraxinifolium Jacq. & 0 & 1 & 0 & 1 & 0 & 0 & 0 & 1 \\
\hline Polypodium polypodioides (L.) Watt. & 0 & 1 & 1 & 1 & 0 & 0 & 1 & 1 \\
\hline PTERIDACEAE & 1 & 0 & 1 & 1 & 1 & 0 & 1 & 1 \\
\hline Adiantopsis radiata (L.) Fée & 1 & 0 & 1 & 0 & 0 & 0 & 1 & 0 \\
\hline Adiantum diogoanum Glaz. ex Baker & 1 & 0 & 1 & 1 & 1 & 0 & 1 & 1 \\
\hline Adiantum latifolium Lam. & 1 & 0 & 1 & 0 & 0 & 0 & 1 & 0 \\
\hline Adiantum raddianum C. Presl & 1 & 0 & 0 & 1 & 0 & 0 & 1 & 1 \\
\hline Adiantum villosum $\mathrm{L}$. & 1 & 0 & 1 & 1 & 0 & 0 & 1 & 0 \\
\hline Doryopteris concolor (Langsd. \& Fisch.) Kuhn & 1 & 0 & 1 & 1 & 0 & 0 & 1 & 0 \\
\hline Pityrogramma calomelanos (L.) Link & 1 & 0 & 0 & 1 & 0 & 0 & 0 & 1 \\
\hline Pteris deflexa Link & 1 & 0 & 1 & 1 & 0 & 0 & 1 & 0 \\
\hline Pteris denticulata Sw. var. denticulata & 1 & 0 & 1 & 1 & 0 & 0 & 1 & 0 \\
\hline Pteris propinqua J. Agardh & 1 & 0 & 0 & 1 & 0 & 0 & 1 & 0 \\
\hline
\end{tabular}


continuação - Tabela 1

continuation - Table 1

\begin{tabular}{|c|c|c|c|c|c|c|c|c|}
\hline Família/Espécie & TE & EP & $\mathrm{MC}$ & FES & FP & $\mathrm{CA}$ & Maravilha & $\begin{array}{l}\text { Capão } \\
\text { da } \\
\text { Várzea }\end{array}$ \\
\hline TECTARIACEAE & 1 & 0 & 1 & 0 & 0 & 0 & 1 & 0 \\
\hline Tectaria incisa Cav. & 1 & 0 & 1 & 0 & 0 & 0 & 1 & 0 \\
\hline THELYPTERIDACEAE & 1 & 0 & 1 & 1 & 1 & 0 & 1 & 1 \\
\hline Thelypteris dentata (Forssk.) E. P. St. John & 1 & 0 & 1 & 1 & 0 & 0 & 1 & 1 \\
\hline Thelypteris dutrai (C.Chr. ex Dutra) Ponce & 1 & 0 & 1 & 1 & 0 & 0 & 1 & 0 \\
\hline Thelypteris hispidula (Decne.) C. F. Reed & 1 & 0 & 1 & 1 & 1 & 0 & 1 & 1 \\
\hline Thelypteris interrupta (Willd.) K. Iwats. & 1 & 0 & 1 & 0 & 1 & 0 & 1 & 0 \\
\hline Thelypteris lugubris (Mett.) R.M.Tryon \& A.F.Tryon & 1 & 0 & 1 & 1 & 0 & 0 & 1 & 0 \\
\hline Thelypteris opposita (Vahl) Ching & 1 & 0 & 0 & 1 & 1 & 0 & 0 & 1 \\
\hline Thelypteris pachyrhachis (Kunze ex Mett.) Ching & 1 & 0 & 0 & 0 & 1 & 0 & 0 & 1 \\
\hline Thelypteris raddii (Rosenst.) Ponce & 1 & 0 & 1 & 1 & 0 & 0 & 1 & 1 \\
\hline Thelypteris rivularioides (Fée) Abbiatti & 1 & 0 & 0 & 0 & 1 & 0 & 0 & 1 \\
\hline Thelypteris salzmanni (Fée) C.V. Morton & 1 & 0 & 0 & 1 & 0 & 0 & 0 & 1 \\
\hline Thelypteris serrata (Cav.) Alston & 1 & 0 & 1 & 1 & 1 & 0 & 1 & 1 \\
\hline \multicolumn{9}{|l|}{ WOODSIACEAE } \\
\hline Diplazium cristatum (Desr.) Alston & 1 & 0 & 1 & 1 & 0 & 0 & 1 & 0 \\
\hline THELYPTERIDACEAE & 1 & 0 & 1 & 1 & 1 & 0 & 1 & 1 \\
\hline Thelypteris dentata (Forssk.) E. P. St. John & 1 & 0 & 1 & 1 & 0 & 0 & 1 & 1 \\
\hline Thelypteris dutrai (C.Chr. ex Dutra) Ponce & 1 & 0 & 1 & 1 & 0 & 0 & 1 & 0 \\
\hline Thelypteris hispidula (Decne.) C. F. Reed & 1 & 0 & 1 & 1 & 1 & 0 & 1 & 1 \\
\hline Thelypteris interrupta (Willd.) K. Iwats. & 1 & 0 & 1 & 0 & 1 & 0 & 1 & 0 \\
\hline Thelypteris lugubris (Mett.) R.M.Tryon \& A.F.Tryon & 1 & 0 & 1 & 1 & 0 & 0 & 1 & 0 \\
\hline Thelypteris opposita (Vahl) Ching & 1 & 0 & 0 & 1 & 1 & 0 & 0 & 1 \\
\hline Thelypteris pachyrhachis (Kunze ex Mett.) Ching & 1 & 0 & 0 & 0 & 1 & 0 & 0 & 1 \\
\hline Thelypteris raddii (Rosenst.) Ponce & 1 & 0 & 1 & 1 & 0 & 0 & 1 & 1 \\
\hline Thelypteris rivularioides (Fée) Abbiatti & 1 & 0 & 0 & 0 & 1 & 0 & 0 & 1 \\
\hline Thelypteris salzmanni (Fée) C.V. Morton & 1 & 0 & 0 & 1 & 0 & 0 & 0 & 1 \\
\hline Thelypteris serrata (Cav.) Alston & 1 & 0 & 1 & 1 & 1 & 0 & 1 & 1 \\
\hline WOODSIACEAE & 1 & 0 & 1 & 1 & 0 & 0 & 1 & 0 \\
\hline Diplazium cristatum (Desr.) Alston & 1 & 0 & 1 & 1 & 0 & 0 & 1 & 0 \\
\hline
\end{tabular}


Na gleba Maravilha, as famílias mais ricas foram Pteridaceae (9), Thelypteridaceae (7), Aspleniaceae (6) e Polypodiaceae (5), enquanto na Capão da Várzea foram Thelypteridaceae (8) e Polypodiaceae (6). Segundo Prado e Hirai (2012), essas famílias estão entre as mais diversas do Estado de São Paulo.

Dados semelhantes sobre as famílias desse estudo foram verificados por Salino (1993; 1996) nas matas ciliares do rio Jacaré-Pepira e na Serra do Cuscuzeiro, em Analândia (SP); no Parque Estadual de Porto Ferreira por Colli et al. (2003); nas glebas Capetinga Leste e Oeste por Colli et al. (2004a, b) no Parque em estudo, e por Nobrega e Prado (2008) no Jardim Botânico Municipal de Bauru (SP), regiões que apresentam vegetação de Mata Ciliar, Floresta Estacional Semidecidual e floresta paludosa.

As famílias Anemiaceae, Lomariopsidaceae, Tectariaceae e Osmundaceae ocorreram apenas na gleba Maravilha, fragmento de Floresta Estacional Semidecidual Montana e Mata Ciliar de inundação temporária, enquanto Cyatheaceae, Dennstaedtiaceae e Osmundaceae somente na Capão da Várzea, onde predomina a Floresta Paludosa.

Do total de espécies deste estudo, 25 foram restritas à Gleba Maravilha, 19 à Capão da Várzea e nove ocorreram em ambas as glebas, quais sejam: Ctenitis submarginalis, Pleopeltis pleopeltifolia, Polypodium polypodioides, Adiantum diogoanum, Adiantum radianum, Thelypteris dentata, Thelypteris hispidula, Thelypteris raddii $\mathrm{e}$ Thelypteris serrata.

A ocorrência de 19 espécies de pteridófitas na Gleba Capão da Várzea, que possui apenas 12,18 hectares, pode ser um indicativo de sua importância para a conservação de espécies típicas de Floresta Paludosa.

Do total de espécies identificadas neste estudo, 35\% ocorreram também no Jardim Botânico Municipal de Bauru (Nobrega e Prado, 2008), onde Blechnum brasiliense, Cyclodium meniscioides, Osmunda regalis,
Thelypteris interrupta, Thelypteris opposita, Thelypteris rivularioides apresentaram-se em ambientes de Floresta Paludosa.

Nenhuma das espécies registradas consta da lista das ameaçadas de extinção do Estado de São Paulo (Mamede et al., 2007).

Os gêneros mais representativos nas glebas Maravilha e Capão da Várzea foram Thelypteris (11) e Asplenium (07), assim como também nos trabalhos realizados no Morro do Cuscuzeiro (Salino, 1996) e no Parque Estadual de Porto Ferreira (Colli et al., 2003).

A maioria das espécies de pteridófitas nas glebas Maravilha e Capão da Várzea possui hábito terrestre. O reduzido número de pteridófitas epífitas foi registrado também nos levantamentos de Salino (1996), Colli et al. (2003), Colli et al. (2004a, b), Colli et al. (2007) e por Nóbrega e Prado (2008) em áreas de vegetação de Floresta Estacional Semidecidual, Mata Ciliar, Cerrado e Floresta Paludosa no interior do Estado de São Paulo, região onde a diversidade de pteridófitas epífitas é menor em relação às florestas ombrófilas do litoral.

As glebas Maravilha e Capão da Várzea são fragmentos florestais que mesmo com área reduzida, isoladas por canaviais e suscetíveis a diversas ações antrópicas conservam juntas aproximadamente $35 \%$ do total de famílias e cerca de $10 \%$ do total de espécies de pteridófitas ocorrentes no Estado de São Paulo.

\section{AGRADECIMENTOS}

Ao Dr. Alexandre Salino pela identificação de materiais botânicos do presente estudo.

Ao Eng. Héverton José Ribeiro, gestor do Parque Estadual de Vassununga na época, pelo apoio no desenvolvimento do trabalho.

Ao Sr. Antonio Crema pelo auxílio durante os trabalhos de campo no Parque. 


\section{REFERÊNCIAS BIBLIOGRÁFICAS}

BARROS, I.C.L.; COSTA e SILVA, M.B. Taxonomia, padrão de venação e aparelhos estomáticos de Pteris schwackeana Christ (Pteridaceae Pteridophyta). Broteria, v. 67, n. 1, p. 257-262, 1996.

COLLI, A.M.T.; SOUZA, S.A. de; SILVA, R.T. da. Pteridófitas do Parque Estadual de Porto Ferreira (SP), Brasil. Rev. Inst. Flor., v. 15, n. 1, p. 29-35, 2003.

et al. Pteridófitas do Parque Estadual da Vassununga, Santa Rita do Passa Quatro (SP), Brasil. Glebas Capetinga Leste e Capetinga Oeste. Rev. Inst. Flor., v. 16, n. 1, p. 25-30, 2004a.

. et al. Pteridófitas do Parque Estadual da Vassununga, Santa Rita do Passa Quatro (SP), Brasil. Gleba Pé-de-Gigante. Rev. Inst. Flor., v. 16, n. 2, p. 121-127, 2004b.

et al. Pteridófitas da Floresta Estadual de Bebedouro, Bebedouro, SP, Brasil. Rev. Inst. Flor., v. 16, n. 2, p. 147-152, 2004c.

et al. Pteridófitas da Reserva Estadual de Águas da Prata, Águas da Prata, SP. Rev. LOGOS, n. 15, p. 11-18, 2007.

MAMEDE, M.C. et al. Livro vermelho das espécies vegetais ameaçadas do Estado de São Paulo. São Paulo: Instituto de Botânica, 2007. 165 p.

NOBREGA, G.A.; PRADO, J. Pteridófitas da vegetação nativa do Jardim Botânico Municipal de Bauru, Estado de São Paulo, Brasil. Hoehnea, v. 1, n. 35, p. 7-55, 2008.

PRADO, J. 1998. Pteridófitas do Estado de São Paulo. In: BICUDO, C.E.M.; SHEPHERD, G.J. (Org.). Biodiversidade do Estado de São Paulo. 2 Fungos Macroscópicos e Plantas. São Paulo: FAPESP. v. 2, p. 47-61.
PRADO, J. As pteridófitas ameaçadas de extinção no Estado de São Paulo. In: MAMEDE et al. (Org.). Livro vermelho das espécies vegetais ameaçadas do Estado de São Paulo. São Paulo: Instituto de Botânica, 2007. p. 39-45.

; SILVESTRE, L.S. As samambaias e licófitas do Brasil. In: FORZZA, R.C. et al. (Org.). Catálogo de plantas e fungos do Brasil. Rio de Janeiro: Jardim Botânico do Rio de Janeiro, 2010. v. 1, p. 69-74.

.; HIRAI, R.Y. Checklist das licófitas e samambais do Estado de São Paulo. Disponível em: $<$ http://biotaneotropica.org.br. $>$. Acesso em: 20 dez. 2012.

RIBEIRO, H.J. (Coord). Plano de Manejo do Parque Estadual de Vassununga. Santa Rita do Passa Quatro, 2009. 328 p. (Em análise no Conselho Estadual de Meio Ambiente CONSEMA).

SALINO, A. Flora pteridofítica das matas ciliares da bacia do rio Jacaré-Pepira, Estado de São Paulo, Brasil. 1993. 277 f. Dissertação (Mestrado em Ciências) - Instituto de Biologia, Universidade Estadual de Campinas, Campinas.

Levantamento das pteridófitas da Serra do Cuscuzeiro, Analândia, SP, Brasil. Revista Brasileira de Botânica, v. 19, n. 2, p. 173-178, 1996.

SÃO PAULO (Estado). Secretaria do Meio Ambiente. Resolução SMA no 48, de 21/9/2004. Disponível em: $<$ http:\|www.cetesb.sp.gov.br/licenciamento/ $1 \mathrm{eg}$ i s 1 a çã o / r e s o 1 u çõ e s / 2004 _ R e s _ SMA_48.pdf $>$. Acesso em: 5 jul. 2006. 
SIQUEIRA, C.R.; WINDISCH, P.G. Pteridófitas da região noroeste do Estado de São Paulo, Brasil: Dennstaedtiaceae. Acta Botanica Brasilica, v. 12, n. 3, p. 357-365, 1998. Suplemento.

WINDISCH, P.G. Pteridófitas da região norte-ocidental do Estado de São Paulo: guia para estudo e excursões. 2. ed. São José do Rio Preto: Universidade Estadual Paulista, 1992. 200 p. 